\title{
Immunological analysis of pesticides: a new tool in groundwater testing
}

\author{
Jens Aamand, Leif Bruun and Claus Bo Vöge Christensen
}

Groundwater is the major source of drinking water in many European countries, and in Denmark alone it accounts for more than $99 \%$ of the drinking water supply. Within the past decade pesticide residues have frequently been detected in groundwater, in many cases at levels exceeding the $0.1 \mu \mathrm{g} / \mathrm{l}$ limit set by the European Community. As a consequence, drinking water abstraction wells have had to be closed in many places in Denmark and other European countries, and a vast amount of money is expended to monitor groundwater pesticide levels. A degradation product of the herbicide dichlobenil, 2,6-dichlorobenzamide (BAM), is the most common cause of drinking water well closure in Denmark. Triazines and their metabolites also contaminate groundwater in many countries, and pose a similar risk to the drinking water supply. Analysis of most pesticides and their degradation products is usually carried out by concentrating the samples by solvent extraction, and identifying the contaminants using gas chromatography (GC) or high-pressure liquid chromatography (HPLC) combined with mass spectrometry (MS). These methods, although robust and well established, are very time-consuming and require specialised instrumentation. The large quantity of solvents used is another draw- back to these methods, as the solvents themselves may be carcinogenic and are also well known contaminants of groundwater. The development of cheap, more sensitive and more rapid pesticide assays is therefore urgent.

Due to their very high sensitivity, immunological methods have long been used in biological science for analysing a large variety of organic structures, but have only recently been introduced to environmental analysis. The benefit of such assays is primarily their high sensitivity, which allows the analysis to be undertaken without the need to concentrate the samples, but also the facility of dealing with large numbers of samples. Compared to conventional analyses, immunological methods face two major drawbacks - one related to specificity and the other to the fact that only very few chemicals can currently be analysed simultaneously. The crux of the specificity problem is that although antibodies react very specifically with particular chemical structures, these same structures may be present in analogous compounds. Thus antibodies developed to recognise, for example the herbicide atrazine might also recognise other triazines (Bruun et al. 2001). An important scientific challenge is therefore the development of highly specific assays recognis-
Fig. 1. Development of monoclonal pesticide antibodies is initiated by covalent conjugation of the pesticide to a carrier protein. This pesticide-carrier complex is injected into mice and after approximately two months the mice have produced antibodies against the pesticide. Selected mice are sacrificed and the spleen is removed to isolate the antibody-producing cells. These cells are difficult to cultivate in vitro, and they are therefore fused with myeloma cells to ensure the viability of the antibody-producing cells. The fused hybridoma cells are cultivated, tested and isolated to achieve monoclonal cultures, which produce one type of antibody only with special characteristics such as binding efficiency and specificity. Slightly modified from Aamand et al. (2003).

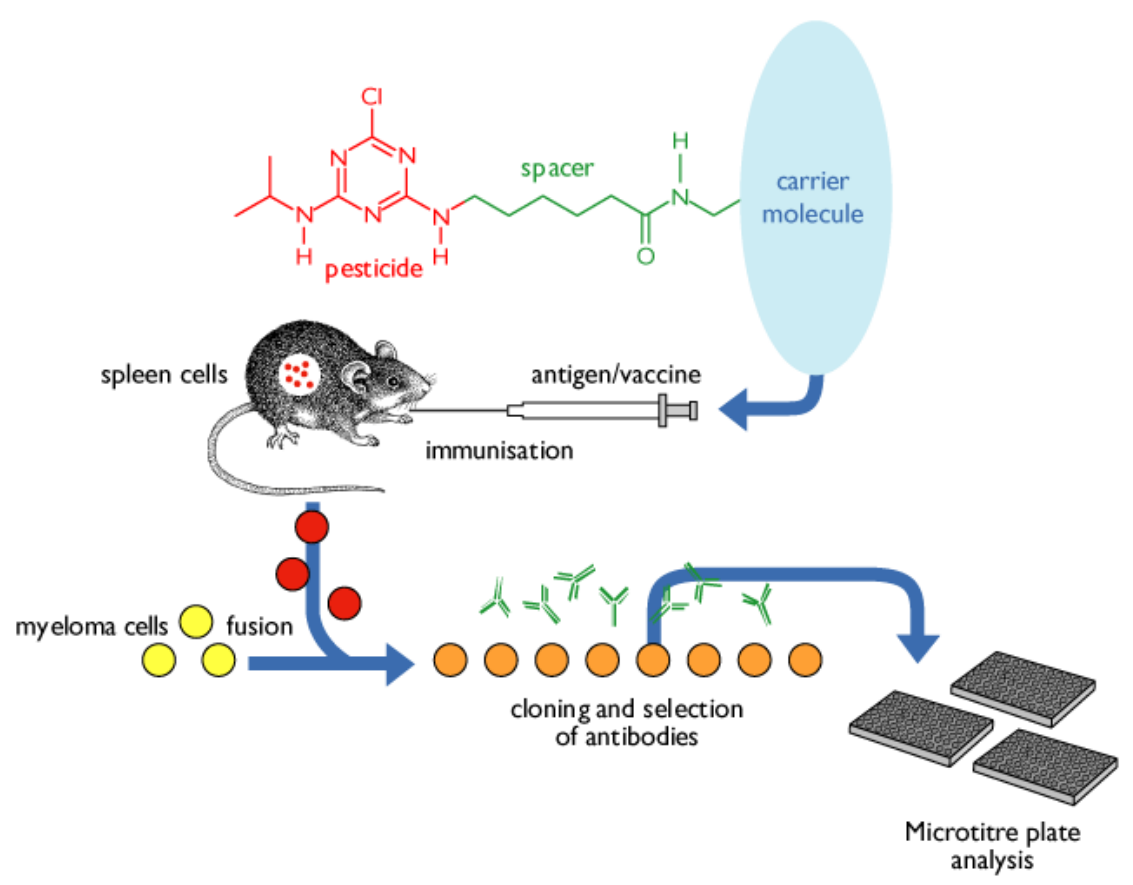




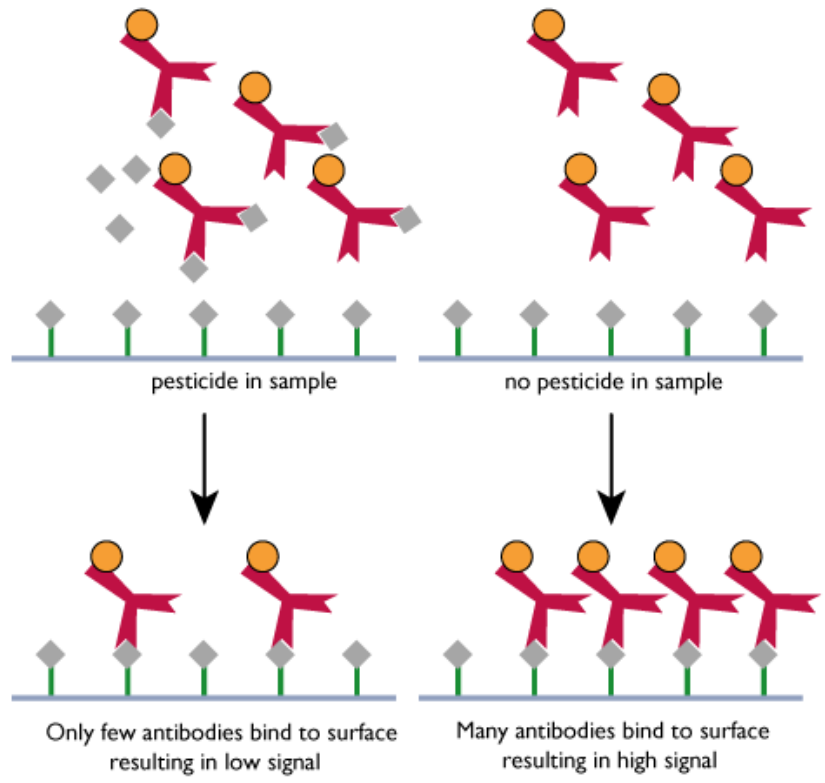

Fig. 2. Example of a competitive assay. In cases with high concentrations of pesticides in the sample, fewer antibodies will bind to the pesticide immobilised to the surface (e.g. the bottom of the microtitre plate) and a low signal will be measured. If the samples do not contain the pesticide, maximal amounts of antibody will bind to the surface resulting in a high signal. Slightly modified from Aamand et al. (2003).

ing each individual compound, as well as assays recognising groups of related chemicals.

With respect to the simultaneous analysis of numerous chemicals, this can be resolved by implementing the new biochip technology, which incorporates the parallellity of sample screening. On a pesticide biochip many specific immunological assays are carried out in isolated small spots on a glass or polymer surface. Each spot has a size of approximately 150 micrometers and forms a specific analysis. Such a miniaturised platform will be usable for monitoring programmes where water samples have to be screened for a range of chemical contaminants.

The overall objectives of this study have been (1) to develop immunoassays for high-sensitivity analysis of specific pesticides and chemically related groups of pesticides, and (2) to transfer the developed assays to a miniaturised biochip platform in a manner allowing analysis of several pesticides simultaneously.

\section{Immunological analysis of pesticides}

The basis for the development of new immunological analyses is the antibody that reacts with complementary molecules, the so-called antigens. Antibodies are part of the immunological defence system in animals and humans for protection against pathogenic vira and bacteria. Following an infection, the organism produces antibodies that recognise and bind to specific molecular structures on the surface of the penetrating bacteria or virus (the antigens). Upon the binding of antibodies, other effector functions of the immune system identify and destroy the bacteria. The chemical structures of the pesticides themselves are too small to induce an immunological response. However, by linking the pesticides to larger carrier molecules it is possible to deceive the immune system into starting the production of antibodies against the pesticide (Fig. 1).

To initiate antibody production the pesticide-carrier complex is injected into an animal, e.g. a rabbit or a mouse, thus inducing an immunological response resulting in the production of antibodies against the pesticide-carrier complex. Antibodies are produced by so-called B-cells each producing a single antibody species, which recognise a specific structure on the pesticide. As the animal contains many B-cells which all produce antibodies, a range of antibodies reacting with different structures on the pesticide and with different affinity will be generated. By purification of the antibodies from the blood serum, a polyclonal antibody serum is obtained, containing antibodies from different B-cells. However, it is often more appropriate to produce monoclonal antibodies (Mab), i.e. specific antibodies all arising from the same B-cell clone.

\section{Production of monoclonal antibodies}

When it has been established by serological screening that the immunised animal produces antibodies with the correct specificity, the spleen, which contains many antibody-producing cells, is removed and grown in culture. Clones producing antibodies with the desired properties are then selected. It is possible to select antibodies that react with chemical structures specific for a single pesticide molecule, or alternatively a structure shared by a group of pesticides such as the triazines.

In addition to their high specificity monoclonal antibodies also have the advantage of consistency. It is always possible to reculture the hybridomas and produce further antibodies with exactly the same characteristics.

\section{Development of immunological assays}

The next step following the selection of suited antibodies is the development of an immunochemical pesticide assay. The analysis is often carried out as a so-called competitive immunoassay in microtitre plates, which are preformed plastic plates with 96 wells (Fig. 1). A known amount of pesticide is immobilised on the bottom of the wells. The samples to be analysed are added to the wells (typically $100 \mu \mathrm{l}$ ), followed by 
the addition of the antibodies. At this point the antibodies can react with either the pesticide immobilised in the microtitre plates or the pesticide in the sample itself. If the concentration of the pesticide in the sample is low, more antibodies will react with the immobilised pesticides and vice versa. When the reaction is completed the microtitre plate wells are washed, leaving only the immobilised pesticideantibody complex (Fig. 2). The antibodies can be directly monitored if coupled with an enzyme, catalysing an enzymesubstrate reaction that yields a coloured end product. The accumulation of the end product is then monitored by absorbance measurements and compared spectrophotometrically to the absorbance of known standards (Fig. 3).

New immunological assays have been developed for several triazines including their degradation products (Bruun et al. 2000a, b, 2001) and for BAM (Bruun et al. 2000c). All assays have a very low detection limit in the range of 0.01-0.02 $\mu \mathrm{g} / \mathrm{l}$, making them ideal for monitoring specific pesticide residues in ground- and drinking water.

Analyses are typically carried out in four replicates, and each microtitre plate also contains a number of pesticide standards. A total of 13 samples can be analysed at each microtitre plate within a period of 3-4 hours.

\section{From microtitre plate to pesticide biochip}

One of the drawbacks of the microtitre format is that it is only possible to analyse for one pesticide in each routine. However, changing the analysis format from microtitre plates to biochips allows for the analysis of several compounds simultaneously. The term 'biochips' describes an analysis where the chemical reactions are not separated by wells, but are carried out on a planar surface such as a glass slide. The principle of the analysis is the same as for the microtitre plates, but the reagents are added as microspots (in the nanoliter range) on the glass surface. Using a robot equipped with printing pins, about 2000 samples/ $\mathrm{cm}^{2}$ can be added as separate spots on the surface. All chemical reactions are then carried out within the individual spots. For the pesticide biochip fluorescence-conjugated monoclonal antibodies were used, which enables the detection of separate signals from each spot on the surface by use of a laser scanner (Fig. 4). As a result of the small dimensions, the individual reactions equilibrate faster and the complete analysis of a biochip can be carried out within 90 minutes. The analyses on the biochips are also more sensitive than on microtitre plates. We have developed a pesticide biochip for BAM and atrazine with a sensitivity of about $1 \mathrm{ng} / \mathrm{l}$ (Fig. 5), which is 100 times less than the limit value for drinking water set by the EU (Belleville et al. 2003, 2004).

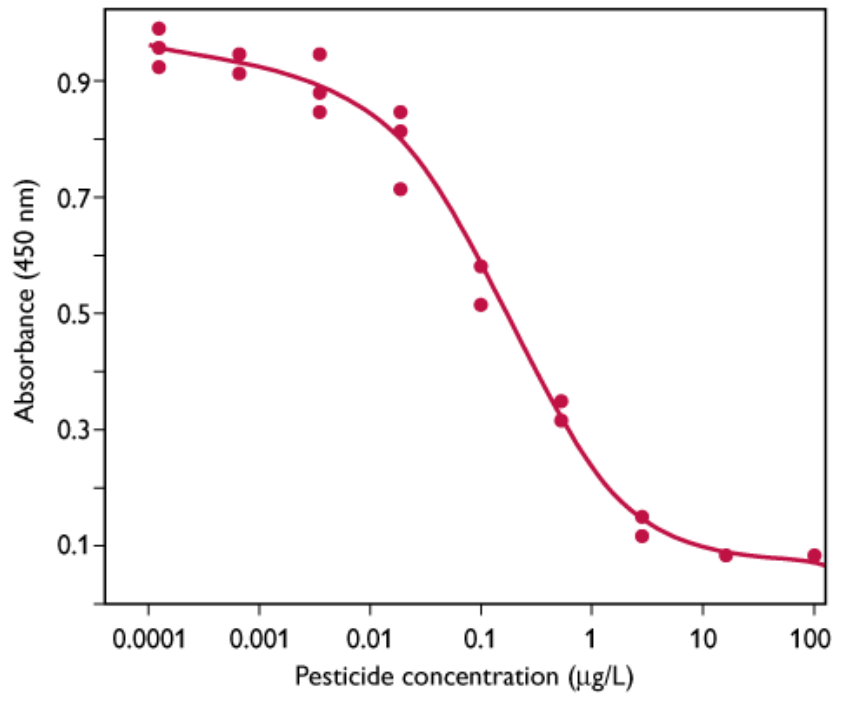

Fig. 3. Example of a standard curve. Note the inverse relationship between pesticide concentration and signal. Slightly modified from Aamand et al. (2003).

\section{Possibilities and limitations}

The benefits of the immunochemical analyses compared to chromatographic techniques are that: (1) less sample volume is needed, which means an easier transport of samples to the laboratory; (2) no solvents or other chemicals are necessary which potentially could pollute the environment; and (3) the immunochemical analyses are much cheaper to carry out.

At present, the immunochemical techniques only enable the analysis of a few compounds simultaneously. In contrast, chromatographic methods (e.g. HPLC or GC/MS) provide the concentration of a range of compounds within the same routine. However, use of the pesticide biochips opens the possibility of analysis of more compounds simultaneously. At present the biochip includes BAM and atrazine only, but in theory it is possible to include additional pesticides as soon as usable antibodies become available.

Another problem to be faced is related to the specificity of the antibodies. Many antibodies may react not only with the targeted pesticide, but also with chemically related compounds. This is the case for the atrazine antibodies that may also react with other triazine herbicides (Bruun et al. 2001). The specificity, however, is not a problem with BAM, because the reactivity of the antibody with other compounds is negligible (Bruun et al. 2000c).

The new pesticide biochip enables the analysis of pesticides in a single drop of water in concentrations as low as $1 \mathrm{ng} / \mathrm{l}$. In principle the pesticide biochip allows the analysis of a range of pesticides, but for the development of such multicomponent analysis further antibodies are needed with high specificities to the individual pesticides. 


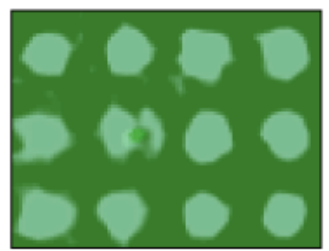

$1 \mathrm{pg} / \mathrm{L}$

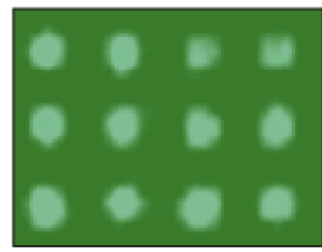

$50 \mathrm{ng} / \mathrm{L}$

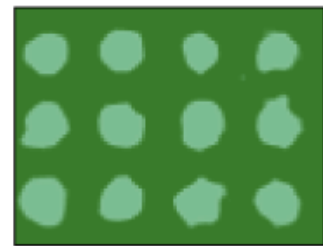

$10 \mathrm{pg} / \mathrm{L}$

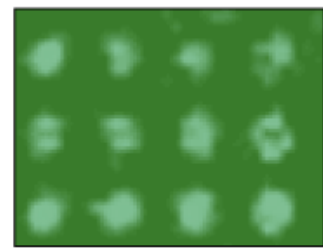

$100 \mathrm{ng} / \mathrm{L}$

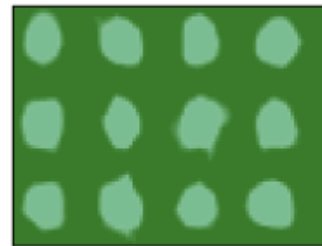

$100 \mathrm{pg} / \mathrm{L}$

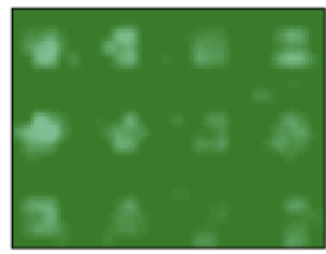

$1 \mu \mathrm{g} / \mathrm{L}$

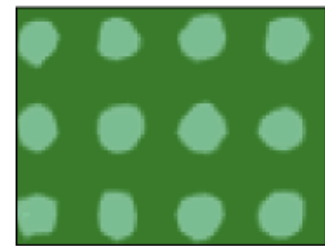

$1 \mathrm{ng} / \mathrm{L}$

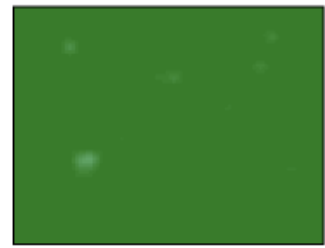

$10 \mu \mathrm{g} / \mathrm{L}$

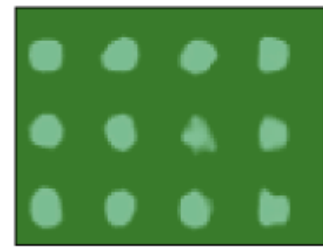

$10 \mathrm{ng} / \mathrm{L}$

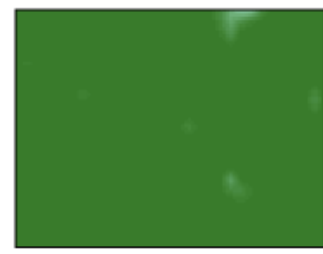

$100 \mu \mathrm{g} / \mathrm{L}$

Fig. 4. Laserscan of a pesticide biochip designed to analyse for BAM and atrazine. Each spot represents a single analysis of a standard with a known concentration. Within each concentration the six spots to the left are BAM and the six spots to the right are atrazine. Slightly modified from Aamand et al. (2003).

\section{Acknowledgement}

The present work is supported by the Immunalyse Project (Grant no. 9901188) financed by the Danish Research Agency.

\section{References}

Aamand, J., Bruun, L. \& Christensen, C.B.V. 2003: Mus hjælper til med pesticidanalyser. Dansk Kemi 84, 29-31.

Belleville, E., Dufva, M., Aamand, J., Bruun, L. \& Christensen, C.B.V. 2003: Quantitative assessment of factors affecting the sensitivity of a competitive immunomicroarray for pesticide detection. BioTechniques 35, 1044-1051.

Belleville, E., Dufva, M., Aamand, J., Bruun, L., Clausen, L. \& Christensen, C.B.V. 2004: Quantitative microarray pesticide analysis. Journal of Immunological Methods 286, 219-229.
Bruun, L., Koch, C., Jakobsen, M.H. \& Aamand, J. 2000a: A new monoclonal antibody for the sensitive detection of cyanazine and other $s$ triazines in water by ELISA. Food and Agricultural Immunology 12(4), 253-262.

Bruun, L., Koch, C., Jakobsen, M.H. \& Aamand, J. 2000b: New monoclonal antibody for the sensitive detection of hydroxy-s-triazines in water by enzyme-linked immunosorbent assay. Analytica Chimica Acta 423, 205-213.

Bruun, L., Koch, C., Pedersen, B., Jakobsen, M.H. \& Aamand, J. 2000c: A quantitative enzyme-linked immunoassay for the detection of 2,6dichlorobenzamide (BAM): a degradation product of the herbicide dichlobenil. Journal of Immunological Methods 240, 133-142.

Bruun, L., Koch, C., Jakobsen, M.H., Pedersen, B., Christiansen, M. \& Aamand, J. 2001: Characterisation of monoclonal antibodies raised against different structures belonging to the $s$-triazine-group of herbicides. Analytica Chimica Acta 436, 87-101.

\section{Authors' addresses}

J.Aa., Geological Survey of Denmark and Greenland, Øster Voldgade 10, DK-1350 Copenhagen K, Denmark. E-mail: jeaa@geus.dk L.B., Statens Serum Institut, Artillerivej 5, DK- 2300 Copenhagen S, Denmark.

C.B.V.C., Technical University of Denmark, Department of Micro- and Nanotechnology, Ørsted Plads 345, DK-2800 Kongens Lyngby, Denmark. 